\title{
EU-Krisenmanagement in Afrika: die Operation EUFOR Tchad/RCA
}

\author{
Hans-Georg Ehrhart*
}

Ein kleines Jubiläum ist zu vermelden: Die Europäische Union führt mit der Entsendung eines Militärkontingents in den Tschad und die Zentralafrikanische Republik (ZAR) ihre zwanzigste Krisenmanagementoperation seit 2003 durch, genannt ,EUFOR Tchad/RCA'. Zum siebten Mal geht es nach Afrika. ${ }^{1}$ Im Kongo engagiert sich die Union seit 2005 mit zwei zivilen Operationen zur Reform der Polizei und des Militärs, nachdem sie dort zweimal militärisch interveniert hat. ${ }^{2}$ Zudem war sie mit einer zivil-militärischen Mission im Sudan engagiert, welche die Afrikanische Union (AU) in ihrem letztlich vergeblichen Bemühen unterstützte, den Völkermord in Darfur zu beenden. Ein Schwerpunkt des Krisenmanagements der Union liegt also offenbar in Afrika. Die Frage ist nur: Warum? Nach eigenem Selbstverständnis betreibt die Europäische Union eine normen- und werteorientierte Außenund Sicherheitspolitik. In der wissenschaftlichen Literatur wird sie denn auch als normative Macht beschrieben, die ihr internationales Umfeld nach allgemein-ethischen und legitimierten Standards gestalten will. ${ }^{3}$ Auch in diesem Beitrag wird normativ in diesem Sinne verstanden. In der internationalen Politik ist hingegen oftmals konsequentialistisches, also interessenorientiertes Handeln dominant. ${ }^{4}$ Das gilt erst recht für den Bereich des Krisenmanagements. Was sind also die Antriebskräfte für dieses Engagement in Afrika im Allgemeinen und in der subsaharischen Krisenregion im Besonderen? Diese Frage soll in mehreren Schritten beantwortet werden. Zunächst werden Konzepte und Ziele des Krisenmanagements in der Afrikapolitik der Europäischen Union sowie die ihnen zugrunde liegenden Werte skizziert. Im Anschluss daran wird die neueste ESVP-Operation in Zentralafrika vorgestellt, bevor in einem weiteren Abschnitt die involvierten Akteure und ihre Interessen analysiert werden. Nach der Erörterung bestehender Probleme und offener Fragen wird abschließend versucht, die Ausgangsfrage nach dem, Warum` zu beantworten.

\section{EU-Krisenmanagement in Afrika: Konzepte, Ziele und normative Grundlagen}

Die normativen Grundlagen der Europäischen Union, die diese zuerst im Innern entwickelt hat, haben eine friedensstiftende Wirkung innerhalb der Europäischen Union entfaltet. Die dabei angewandten Methoden der Integration, Kooperation und Verflechtung gründen auf gesellschaftlichen Werten wie Freiheit, Demokratie, und Rechtsstaatlichkeit sowie der Achtung der Menschenrechte und Grundfreiheiten, die mit zunehmender außen- und sicherheitspoli-

* Dr. Hans-Georg Ehrhart, Senior Research Fellow und stellvertretender Leiter, Zentrum für Europäische Friedens- und Sicherheitsstudien am Institut für Friedensforschung und Sicherheitspolitik an der Universität Hamburg.

1 Bei dieser Zählung wird EUPOL RD Congo als Nachfolgeoperation von EUPOL Kinshasa als neue Operation gezählt. Vgl. die Internetseite des Rats der Europäischen Union, abrufbar unter: http://www.consilium.europa.eu (letzter Zugriff: 12.03.2008).

2 Vgl. Hans-Georg Ehrhart: Zwischen rationalistischem Kalkül und Logik der Angemessenheit: Zur Ethik des Befriedungskonzepts von VN und EU im Kongo, in: Sicherheit und Frieden (S+F) 3/2007, S. 105-111.

3 Vgl. Ian Manners: Normative Power Europe Reconsidered: beyond the Crossroads, in: Journal of European Public Policy 1/2006, S. 182-199 und Nathalie Tocci: Profiling Normative Foreign Policy: The European Union and its Global Partners, CEPS Working Document 279/2007.

4 Vgl. dazu James G. March/Johan P. Olson: The Logic of Appropriateness, ARENA Working Papers 4/09. 
tischer Handlungsfähigkeit auch auf internationaler Ebene gestärkt werden sollen. Als Ziele der Gemeinsamen Außen- und Sicherheitspolitik (GASP) formuliert Artikel 11 EU-Vertrag dementsprechend die Wahrung der gemeinsamen Werte und Interessen im Einklang mit den Grundsätzen der UN-Charta, Wahrung des Friedens und die Stärkung der internationalen Sicherheit, die Förderung der internationalen Zusammenarbeit, die Entwicklung und Stärkung von Demokratie und Rechtsstaatlichkeit sowie die Achtung der Menschenrechte und Grundfreiheiten.

In der Europäischen Sicherheitsstrategie (ESS) geht die Union von einem weiten Sicherheitsverständnis aus. ${ }^{5}$ Dieses gründet zunächst auf der Annahme, dass sich Sicherheit und Entwicklung gegenseitig bedingen. Interventionsfähigkeit unter Einschluss militärischer Mittel wird als notwendige Voraussetzung für die Bewältigung globaler Herausforderungen und Bedrohungen angesehen. Gemäß der ESS sind die Hauptbedrohungen der transnationale Terrorismus, die Proliferation von Massenvernichtungswaffen, regionale Konflikte, Staatszerfall und die organisierte Kriminalität. Die Europäische Union fühlt sich bei ihren Gegenmaßnahmen einem wirksamen Multilateralismus auf der Grundlage der UN-Charta und der darin enthaltenen transnationalen Normen verpflichtet. Darüber hinaus sichert sie zu, die Vereinten Nationen zu stärken. Deren Ausstattung mit den für ein effizientes Handeln erforderlichen Mitteln sei für Europa ein vorrangiges Ziel.

Dieser Ansatz wurde Anfang 2004 durch den ,Gemeinsamen Standpunkt im Hinblick auf die Vermeidung, Bewältigung und Beilegung von Konflikten in Afrika' weiter ausgeführt. Als Ziele werden hier genannt: die Stärkung afrikanischer Einsatzmittel und Fähigkeiten, die enge Zusammenarbeit mit der UNO und regionalen Organisationen sowie die Bereitschaft der Europäischen Union, sich ,wann immer erforderlich“ bei der Krisenbewältigung in Afrika mit eigenen Mitteln zu engagieren. ${ }^{6}$ Mit der Verabschiedung eines ESVP-Aktionsplans für Afrika zehn Monate später erfolgte ein erster Schritt zur Umsetzung der gemeinsamen Position. Er enthielt konkrete Maßnahmen wie technische Beratung im Bereich Operationsplanung und -führung, Ernennung von Verbindungsoffizieren, Aufstellung von Expertenteams für die Sicherheitssektorreform und Durchführung von Trainingskursen. ${ }^{7}$

Am 19. Dezember 2005 verabschiedete der Europäische Rat eine Afrikastrategie, die folgende Themen behandelt: Frieden und Sicherheit, Menschenrechte und Regierungsführung, Entwicklungshilfe, nachhaltiges Wachstum, regionale Integration und Handel sowie Förderung von Humankapital. Im friedens- und sicherheitspolitischen Bereich bekräftigt die Europäische Union ihre Absicht, mit den afrikanischen Partnern bei der Konfliktprävention, Konfliktbearbeitung und Friedenserhaltung zusammenzuarbeiten sowie Frieden und Stabilität durch direkte Unterstützung im Rahmen der Europäischen Sicherheits- und Verteidigungspolitik (ESVP) zu fördern und die Unterstützungsmaßnahmen für die Friedenskonsolidierung in Afrika zu intensivieren. Zudem will sie die Zusammenarbeit im Kampf gegen den illegalen Waffenhandel und den internationalen Terrorismus verstärken. ${ }^{8}$ Knapp ein Jahr später verabschiedete der Rat das EU-Konzept zur Stärkung afrikanischer Fähigkeiten für

5 Vgl. Europäischer Rat: Ein sicheres Europa in einer besseren Welt, Europäische Sicherheitsstrategie, Brüssel 2003.

6 Vgl. Gemeinsamer Standpunkt 2004/85/GASP des Rates vom 26. Januar 2004 im Hinblick auf die Vermeidung, Bewältigung und Beilegung von Konflikten in Afrika und zur Aufhebung des Gemeinsamen Standpunkts 2001/374/GASP, in: Amtsblatt der Europäischen Union, Nr. L 21 vom 28. Januar 2004, S. 25-29, hier S. 25.

7 Vgl. Rat der Europäischen Union: Action Plan for ESDP support to Peace and Security in Africa, 10538/4/04 REV 4, 16. November 2004.

8 Vgl. Rat der Europäischen Union: The EU and Africa. Towards a Strategic Partnership, 15961/05 (Presse 367), 19. Dezember 2005. 
die Verhütung, Bewältigung und Beilegung von Konflikten. Es soll kohärente und umfassende EU-Rahmenvorgaben für die Verwirklichung der Kernpunkte der EU-Strategie für Afrika im Bereich Frieden und Sicherheit bieten. ${ }^{9}$

Ende 2007 gelang es auf dem zweiten Gipfeltreffen mit der AU schließlich ein Dokument zur Begründung einer Strategischen Partnerschaft zu verabschieden. Eines der vier Hauptziele besteht darin, Frieden und Sicherheit sowie demokratische Regierungsführung und Menschenrechte ,as preconditions for political, economic and social development“ zu fördern. ${ }^{10}$ Der Aktionsplan zur Umsetzung der Strategischen Partnerschaft definiert für den Bereich Sicherheit und Frieden drei vorrangige Aktionsbereiche: Verstärkung des Dialogs über friedens- und sicherheitspolitische Herausforderungen, vollständige Verwirklichung der afrikanischen Friedens- und Sicherheitsarchitektur und gesicherte Finanzierung afrikanisch geführter Friedensoperationen. ${ }^{11}$

Die Europäische Union hat also in den letzten Jahren ihre allgemeinen Zielsetzungen für die GASP hinsichtlich ihrer Politik gegenüber dem afrikanischen Kontinent in mehreren konzeptionellen Schritten näher bestimmt und auf höchster Ebene politisch beschlossen. Sie verfolgt demnach sogenannte Milieuziele, ${ }^{12}$ in deren Mittelpunkt die Stärkung afrikanischer Fähigkeiten steht („African ownership“). Die Umsetzung dieses politisch-strategischen Ansatzes erfolgte im Bereich der Konfliktbearbeitung unter anderem durch zivile und militärische ESVP-Missionen. Dabei wird der Reform des Sicherheitssektors eine große Bedeutung beigemessen. So ist die Entsendung einer entsprechenden Mission nach Guinea-Bissau vorgesehen. ${ }^{13}$ Ein weiterer Schwerpunkt liegt auf der Stärkung afrikanischer Kapazitäten zur Konfliktbearbeitung durch Training, Beratung, technische Hilfe und logistische Unterstützung. Dementsprechend unterstützt die Europäische Union den Aufbau einer afrikanischen Friedenstruppe durch die AU. ${ }^{14}$ Ein wesentliches Instrument ist die Afrikanische Friedensfazilität (APF), die für den Zeitraum von 2008-2010 auf 300 Millionen Euro erhöht worden ist. Sie ist die wichtigste Finanzierungsquelle für den Aufbau afrikanischer Friedensstrukturen und die Durchführung entsprechender Aktivitäten. ${ }^{15}$

\section{EUFOR Tchad/RCA: Hintergrund und Aufgaben}

Nun geht es in den Osten des Tschad und den Nordosten der Zentralafrikanischen Republik, also in jene Nachbarländer des Sudan, die eine gemeinsame Grenze mit der Krisenregion

9 Vgl. Rat der Europäischen Union: Mitteilung an die Presse, 2760. Tagung des Rates Allgemeine Angelegenheiten und Außenbeziehungen: Allgemeine Angelegenheiten, 14779/06 (Presse 302), 13. November 2006.

10 The Africa-EU Strategic Partnership. A Joint Africa-EU Strategy, S. 4, abrufbar unter: http://www.eu2007.pt/ UE/vEN/Noticias_Documentos/20071209PARCEST.htm (letzter Zugriff: 29.01.2008).

11 Vgl. ebenda, S. 30-34.

12 Nach Arnold Wolfers sind „Milieu Goals“ im Unterschied zu „Possession Goals“ nur indirekt mit Eigeninteressen verbunden. Sie gelten längerfristig und ihr Schwerpunkt liegt auf der regelbasierten Gestaltung des internationalen Umfeldes. Vgl. Nathalie Tocci: Profiling Normative Foreign Policy, 2007, S. 3-4.

13 Es handelt sich um die Mission EU SSR Guinea-Bissau. Vgl. die Internetseite der Europäischen Union, abrufbar unter: http://www.consilium.europa.eu/ (letzter Zugriff: 13.03.2008).

14 Eine Maßnahme in diesem Kontext ist die Europäisierung des französischen Programms RECAMP. Es zielt auf die Ausbildung ziviler und militärischer Expertise für Friedensmissionen im Hinblick auf die für 2010 vorgesehene Aufstellung einer afrikanischen Brigade von 5.000 Einsatzkräften. Vgl. Au coeur du patenariat stratégique: EURO RECAMP, in: Rat der Europäischen Union: ESDP Newsletter 5/2007, S. 20.

15 Diese Budgetlinie wurde 2004 auf Bitten der AU eingeführt und umfasste ursprünglich 250 Millionen Euro für den Aufbau kollektiver Friedens- und Sicherheitsstrukturen und -aktivitäten in Afrika. 242 Millionen Euro wurden bislang für die Unterstützung von Friedenseinsätzen der AU oder subregionaler Organisationen ausgegeben, 35 Millionen Euro für den Aufbau von Strukturen. Vgl. William Assanvo/Christian E. B. Pout: The European Union (EU): African Peace and Security Environment's Champion?, in: Fondation pour la Recherche Stratégique (Hrsg.): Points de Vue, 27. November 2007. 
Darfur haben. In der Region schien sich letztes Jahr ein ,Fenster der Möglichkeiten` zu öffnen: Im Juli 2007 wurde die UNO/AU-Hybridoperation für Darfur (UNAMID) beschlossen und zwei Monate später die multi-dimensionale UN-Mission im Tschad und in der ZAR (MINURCAT). ${ }^{16}$ Im Tschad begann im Februar 2007 ein interner politischer Dialog, der zu Wahlen im Jahr 2009 führen sollte. Ende Oktober 2007 fand in Libyen eine von der UNO und der AU organisierte Friedenskonferenz über Darfur statt, die in einen graduellen Friedensprozess münden sollte. Beide Prozesse sind jedoch extrem gefährdet. Zum einen fanden im Tschad wieder heftige Kämpfe zwischen Regierung und Rebellen statt, in deren Verlauf Mitglieder der politischen Opposition verhaftet wurden. Zum anderen waren die Ergebnisse der Friedenkonferenz bislang sehr enttäuschend.

Das strategische Ziel der Europäischen Union besteht darin, mit ihren politischen, humanitären und sicherheitspolitischen Mitteln zu einer Lösung des Darfur-Konflikts beizutragen, um eine Verschärfung oder ein Übergreifen auf angrenzende Gebiete zu verhindern. Sie will UNAMID unterstützen, die seit dem 31. Dezember 2007 die weitgehend erfolglose Mission der AU im Sudan (AMIS) abgelöst hat. Zudem will die Europäische Union durch die Entsendung eines Einsatzverbandes von bis zu 4.000 Soldaten an der Stabilisierung der an Darfur angrenzenden Regionen mitwirken, der spätesten nach einem Jahr von einer UNOBlauhelmtruppe abgelöst werden soll. Es handelt sich also um eine sogenannte ,Überbrückungsoperation', wie sie die Europäische Union bereits 2003 für knapp vier Monate im Osten des Kongo durchgeführt hat. ${ }^{17}$ Sie ergänzt die Mission MINURCAT, deren Aufgabe darin besteht, ,to help create the security conditions conducive to a voluntary, secure and sustainable return of refugees and displaced persons, inter alia by contributing to the protection of refugees, displaced persons and civilians in danger, by facilitating the provision of humanitarian assistance in eastern Chad and the north-eastern Central African Republic and by creating favourable conditions for the reconstruction and economic and social development of those areas". ${ }^{18}$

Hauptaufgabe des Militärkontingents ist es, zu einem stabilen Sicherheitsumfeld im Osten des Tschad und im Nordosten der Zentralafrikanischen Republik beizutragen. So soll EUFOR die Arbeit der UN-Polizei und der seit Langem dort tätigen humanitären Organisationen gewährleisten und die Voraussetzungen für die Rückkehr der vertriebenen Bevölkerung in ihre Heimatregionen verbessern. Keine leichte Aufgabe, wenn man bedenkt, dass sich im Tschad 234.000 Flüchtlinge aus Darfur, 41.000 aus der Zentralafrikanischen Republik und 179.000 Binnenvertriebene sowie innerhalb der ZAR 3.000 Flüchtlinge aus Darfur und 20.000 Binnenflüchtlinge in und außerhalb der zahlreichen Lager aufhalten, wo sie Übergriffen der verschiedenen Kriegsparteien oder marodierender Banden ausgesetzt sind. ${ }^{19}$

Die Europäische Union kann einerseits an ihr bisheriges Engagement in der Region anknüpfen. Dieses reicht von humanitären und entwicklungspolitischen Maßnahmen in beiden Ländern über die Förderung von Wahlen im Tschad bis hin zur Finanzierung der Multinationalen Streitkraft in der Zentralafrikanischen Republik (FOMUC). Geplant sind ferner Maßnah-

16 MINURCAT soll 300 Polizisten, 50 Verbindungsoffiziere und eine angemessene Zahl von zivilem Personal umfassen. Vgl. UN-Sicherheitsrat: Resolution 1778 (2007), adopted by the Security Council at its 5748th meeting, on 25 September 2007, S/RES/1778 (2007), 25. September 2007, S. 3.

17 Bei der im Jahre 2006 durchgeführten EUFOR RD Congo handelte es sich um eine „Stand-by-Operation“. Der Einsatzverband hatte die primäre Aufgabe, Störer der ersten freien Wahlen nach über vierzig Jahren abzuschrecken und die UNO-Blauhelmtruppe MONUC zu unterstützen.

18 Abrufbar unter: http://www.un.org/Depts/dpko/missions/minurcat/mandate.html (letzter Zugriff: 31.01.2008).

19 Vgl. Central Intelligence Agency: The 2008 World Factbook, abrufbar unter: https://www.cia.gov/library/publications/the-world-factbook/geos/cd.html (letzter Zugriff: 26.01.2008) und Le Monde: L'Union européenne lance l'opération Eufor au Tchad et en Centrafrique, 30.01.2008. 
men zur Reform des Militärs und der Polizei. ${ }^{20}$ Andererseits muss sie auf politische Sensibilitäten der betroffenen Länder Rücksicht nehmen. Darum sieht sie sich in ihrem Krisenmanagementkonzept und angesichts ihrer Möglichkeiten zu Einschränkungen genötigt, die problematisch sind und sogar den Erfolg der Mission infrage stellen könnten. So soll die Kontrolle der Grenze nicht zum Mandat gehören, sondern unter ausschließlich nationaler Verantwortung bleiben, obwohl die grenzüberschreitenden Bewegungen bewaffneter Gruppen ein Kernproblem sind. Des Weiteren soll das EU-Kontingent nicht in Flüchtlingslagern und im unmittelbaren Umfeld eingreifen, obwohl nicht gewiss ist, ob die dafür vorgesehenen tschadischen und UNO-Polizeikräfte dazu in der Lage sind. Schließlich soll sich das Kontingent nicht in die inneren Angelegenheiten der beiden Länder einmischen, obwohl deren Sicherheitskräfte für die Flüchtlinge und für die eigene Bevölkerung ein Teil des Sicherheitsproblems darstellen.

\section{Akteure und Interessen}

Problematisch sind auch die vielfältigen und zum Teil gegensätzlichen Interessen der involvierten Akteure, welche die Europäische Union in ihr politisches Kalkül einbeziehen muss. Da ist zum einen Präsident Idriss Déby, der sich 1990 mit sudanesischer und französischer Unterstützung im Tschad an die Macht putschte und sich seitdem mit einer Mischung aus Unterdrückungsmaßnahmen, Intrigen und Demokratisierungsversprechen dort hält. Er setzt weiterhin auf eine Kultur der Gewalt, die seit der Staatsgründung auf dem Land lastet. Déby geht es in erster Linie um den Erhalt der Macht. Um diese zu sichern hat er die Verfassung ändern und Wahlen fälschen lassen. Seit der Tschad Ölexporteur ist, hat Déby das ursprünglich vorbildliche Abkommen mit der Weltbank über die Verwendung der Einkünfte aus dem Energiesektor für die Entwicklung des Landes einseitig verändert. Er finanziert mit diesen Einnahmen nun Waffen für die Aufrüstung seiner Armee und substaatlicher Akteure. Dazu gehören auch Rebellengruppen, die sich aus dem im Tschad und in Darfur siedelnden Stamm der Zaghawa rekrutieren, dem Déby selbst angehört. ${ }^{21}$ Diese Politik brachte ihn in Konflikt mit dem Sudan, seitdem er Rebellenbewegungen unterstützt, die in Darfur gegen das Regime in Khartum kämpfen.

Der Sudan zahlt mit gleicher Münze zurück. Er unterstützt Regimegegner innerhalb des Tschad und die arabischen Reitermilizen Janjaweed, die mittlerweile nicht nur in Darfur ihr blutiges Unwesen treiben, sondern auch im Nachbarland. Bemühungen, diesen Stellvertreterkrieg zu beenden, sind bislang gescheitert, obwohl Libyen, Eritrea und Saudi-Arabien, die AU und die UNO um Vermittlung bemüht sind und die Hauptprotagonisten sich zum wiederholten Mal verpflichtet haben, die Rebellengruppen im jeweils anderen Lande nicht mehr zu unterstützen. ${ }^{22}$ Der prekäre Frieden zwischen dem islamischen Norden und dem animis-

20 Die Europäische Kommission will 2008 folgende Maßnahmen im Einsatzgebiet finanzieren: humanitäre Hilfe (38,5 Mio.), Sicherheitssektorreform (10 Mio.), Wiederaufbau und Eingliederungshilfen (13 Mio.). Vgl. die Internetseite der Vertretung der Europäischen Union bei den Vereinten Nationen, abrufbar unter: http:// www.eu-un.europa.eu (letzter Zugriff: 09.10.2007).

21 Die Zaghawa stellen drei Prozent der Bevölkerung, bekleiden aber 80 Prozent der Führungspositionen bei den Sicherheitskräften. Sie bilden keine monolithische Einheit. Die Clans konkurrieren vielmehr miteinander, was in jüngster Zeit zu Rebellionen und Desertionen selbst engster Familienmitglieder geführt hat. Eine andere große Rebellengruppe will nicht nur die Vorherrschaft Débys beenden, sondern der Zaghawa. Durch zahlreiche Abspaltungen und unterschiedliche Strategien ist die bewaffnete Opposition gegen Déby allerdings geschwächt. Vgl. Volker Gerdesmeier: Gewaltsame Konflikte im Tschad. Ursachen, Akteure und Handlungsoptionen, FriEnt/Misereor Konfliktanalyse, Bonn/Aachen, Dezember 2007.

22 Das jüngste dieser Abkommen wurde am 13. März 2008 am Rande der Tagung der Organisation der Islamischen Konferenz unter Beisein von UNO Generalsekretär Ban Ki-moon geschlossen. Im Gegensatz zu früheren Vereinbarungen ist die Einrichtung einer internationalen Kontaktgruppe und eines monatlichen Monitoring vorgesehen. Die Rebellengruppen in beiden Ländern sprachen sich gegen das Abkommen aus, weil sie nicht konsultiert wurden. Damit schwindet die Hoffnung, dass dieser Vertrag gehalten wird. Vgl. Le Monde: Nouvel accord de paix entre N'Djamena et Khartoum, 15.03.2008. 
tischen Süden des Landes sowie die politische Rückendeckung durch China scheinen es dem in die OPEC strebenden Sudan gegenwärtig zu erlauben, im Darfurkonflikt kompromisslos zu bleiben. ${ }^{23}$ So soll Khartum auch hinter dem gescheiterten Umsturzversuch im Tschad von Anfang Februar 2008 stehen. Die Konflikte in Darfur und im Tschad sind also eng miteinander verknüpft. Aber auch der sudanesische Nord-Süd-Konflikt spielt eine Rolle. Im Sudan sind zwar für 2009 Wahlen vorgesehen, die die derzeitige Interimsregierung beenden sollen. Allerdings beschuldigen sich die ehemaligen Konfliktparteien gegenseitig, das Friedensabkommen von 2005, das auch eine Volksabstimmung über die Unabhängigkeit des Südsudan 2011 vorsieht, zu hintertreiben. ${ }^{24}$ Es besteht also die Gefahr, dass ein halbwegs eingedämmter Konflikt wieder ausbricht und - vor dem Hintergrund der jüngsten blutigen Ereignisse in Kenia und am Horn von Afrika - große Teile Subsahara-Afrikas in Brand geraten. ${ }^{25}$

Der Präsident der ZAR, François Bozizé, putschte sich 2003 an die Macht und wurde 2005 durch Präsidentschafts- und Parlamentswahlen in seinem Amt bestätigt. Er wird politisch und militärisch von Frankreich, dem Tschad und der Zentralafrikanischen Wirtschafts- und Währungsunion unterstützt, die Soldaten für die FOMUC stellt. Er befürwortet einen inklusiven politischen Dialog, doch erst wenn sich die Sicherheitslage verbessert. Diese Forderung zielt auf die angebliche Unterstützung der zentralafrikanischen Rebellenbewegung UFDR durch den Sudan.

Libyen betrachtet den Tschad als seine natürliche Einflusssphäre. In den 1980er Jahren verkündete Staatsoberhaupt Muammar al-Gaddafi schon einmal die Vereinigung beider Länder. Er führte aber auch jahrelang Krieg gegen den Tschad, dessen Armee von Frankreich und den USA unterstützt wurde. Seit der außenpolitischen Wende Libyens, in deren Verlauf er auch sein Streben nach Nuklearwaffen aufgab, neigt Gaddafi zu einer vermittelnden bis Déby unterstützenden Haltung im Tschad-Konflikt. So war er eine der treibenden Kräfte für die Durchführung der Darfur-Friedenskonferenz im Oktober 2007. Während des gewaltsamen Umsturzversuches Anfang Februar 2008 stellte er (auf französisches Ersuchen) Munition für die tschadischen T-55Panzer zur Verfügung und kritisierte das Vorgehen der Rebellen gegen die legitime Regierung. ${ }^{26}$ Die AU ernannte ihn dennoch zum Vermittler im Konflikt zwischen Tschad und Sudan.

Frankreich ist der langjährige Schutzpatron Bozizés und Débys. Der Präsident des Tschad gilt als Stabilitätsfaktor für das politisch und ethnisch zerrissene Land. Dabei spielen historische Bindungen ebenso eine Rolle wie geostrategische und wirtschaftliche Interessen. Paris hat seiner interventionistischen Politik in Afrika abgeschworen und sein militärisches Engagement auf dem schwarzen Kontinent dementsprechend reduziert. ${ }^{27}$ Gleichzeitig ver-

23 Vgl. International Herald Tribune: Once again, Sudan shows its scorn for the world, 23.01.2008.

24 Die Umsetzung des umfassenden Friedensabkommens für den Sudan hinkt weit hinter dem Zeitplan her. Khartum fürchtet eine wachsende Popularität der südsudanesischen Volksbefreiungsbewegung SPLM und eine Abspaltung des ressourcenreichen Südens, während dieser gerade das anstrebt. Indem Khartum den heißen Darfurmit dem schwelenden Nord-Süd-Konflikt verknüpft, verzögert es die Umsetzung des Friedensabkommens.

25 Vgl. zur Lage im Sudan die Internetseite der International Crisis Group, abrufbar unter: www.crisisgroup.org (letzter Zugriff: 12.03.2008). Vgl. auch Zentrum für Internationale Friedenseinsätze: Asian, European and African Policies, Practices and Lessons Learnt in Peace Operations in Africa. DR Congo, Sudan and the Darfur Conflict. An Indian - European Dialogue in the Context of the German EU Presidency 2007, Report 08/2007, S. 8-14.

26 Vgl. Philippe Bernard/Natalie Nougayrède: Tchad: Paris avait décidé de ne pas intervenir, in: Le Monde, 08.02.2008.

27 Damit ist keine grundsätzliche Absage an eine militärisch instrumentierte Weltpolitik verbunden, sondern eher eine inhaltliche und geografische Umorientierung. Präsident Sakorzy will die französischen Streitkräfte nur noch mit einem Mandat der AU oder der UNO in Afrika einsetzen. Frankreich und die Vereinigten Arabischen Emirate haben die Errichtung eines Militärstützpunktes an der Meerenge von Hormus direkt gegenüber dem Iran und in der Nähe eines amerikanischen Stützpunktes in Bahrein vereinbart. 400 Soldaten sollen dort stationiert werden. Es ist die erste ständige Militärbasis Frankreichs am Persischen Golf und das erste Mal, dass ein solcher Stützpunkt in einem Land errichtet wird, mit dem Paris keine koloniale Vergangenheit hat. Vgl. Frankfurter Allgemeine Zeitung: Französische Basis in Abu Dhabi, 16.01.2008. 
sucht es jedoch seine Afrikapolitik zu europäisieren, womit auch die Hoffnung auf ein stärkeres deutsches Engagement verbunden ist. ${ }^{28}$ Das bringt Legitimation, spart Kosten und schafft Einfluss. Zugleich bleibt Frankreich mit mehreren afrikanischen Staaten über Verteidigungsabkommen verbunden. Auf der Basis solcher bilateralen Abkommen sind zurzeit 1.100 Soldaten im Tschad und 420 in der ZAR stationiert. Die ,Opération Epervier' genannte Militärpräsenz im Tschad hat seit 1986 den offiziellen Auftrag, die Souveränität des Landes zu verteidigen, während die 2002 begonnene ,Opération Boali“ in der Zentralafrikanischen Republik die FOMUC und die nationale Armee unterstützt. ${ }^{29}$ EUFOR Tchad/RCA hat einen anderen Auftrag und eine andere Führungsstruktur. Paris (und seinen EUFORPartnern) ist sehr an der Verdeutlichung dieses Unterschieds gelegen. Gleichwohl dürfte er nicht für alle leicht nachvollziehbar sein, denn EUFOR wird vom französischen Kontingent im Tschad personell, logistisch und mit Aufklärungskapazitäten unterstützt. ${ }^{30}$ Trotz dieser Komplikation lancierten und unterstützen Präsident Sarkozy und Außenminister Kouchner als Mitgründer von „Ärzte ohne Grenzen“ einer der frühen Befürworter humanitärer Interventionen - EUFOR vor allem aus humanitären und sicherheitspolitischen Gründen. Die durch die jüngsten Gewaltakte verschärfte Flüchtlingsmisere im Tschad und in der ZAR erhöht die Instabilität in Ländern, die zu den ärmsten und korruptesten der Welt gehören. Paris fürchtet letztlich eine ,Somalisierung' der gesamten Region, sodass es auf verschiedenen Ebenen - UNO, Europäische Union, bilateral - aktiv ist, um den Krisenherd einzudämmen.

Es weiß sich dabei an der Seite der USA, die ihrerseits mehrere Interessen im Tschad verfolgen: Die Mineralölkonzerne Exxon und Chevron sind führend an der Ausbeutung des Ölfeldes Doba beteiligt. Die nationale Sicherheitsstrategie der USA aus dem Jahre $2002 \mathrm{stuft}$ Subsahara-Afrika als energie- und sicherheitspolitisch bedeutend ein. Washington sieht im Tschad einen strategischen Alliierten im Kampf gegen den islamischen Fundamentalismus. ${ }^{31}$ Zudem sieht es sich im zunehmenden Konkurrenzkampf mit China um afrikanische Energiequellen. Des Weiteren befürchtet es, im Falle einer Verschlimmerung der Lage im Krisengebiet Sudan/Tschad/ZAR unter erhöhten Handlungsdruck zu geraten - zu einer Zeit, da seine militärischen Kräfte voll ausgelastet sind. Gleichwohl verstärkt Washington seine militärischen Aktivitäten auf dem bis zum 11. September 2001 lange vernachlässigten Kontinent. So unterhält es in Dschibuti seinen einzigen permanenten Stützpunkt in Afrika. Washington hat darüber hinaus mit verschiedenen Staaten Vereinbarungen über sogenannte access points für US-Streitkräfte in Krisenfällen vereinbart. Im Oktober 2007 nahm zudem das neue Regionalkommando für Afrika (AFRICOM) seine Arbeit auf. ${ }^{32}$

28 Vgl. Jean-Marc Châtaigner: Zwischen Reform und Tradition: Die französische Afrikapolitik heute, Hintergrundinformation aus der internationalen Entwicklungszusammenarbeit, Friedrich-Ebert-Stiftung, Oktober 2006.

29 Vgl. Linternaute Magazine: Les opérations extérieures de l'armée française. Tchad: 1.100 soldats français, abrufbar unter: http://www.linternaute.com/actualite/savoir/06/armee-francaise-etranger/tchad.shtml (letzter Zugriff: 28.01.2008).

30 Vgl. Philippe Bernard: La mission de l'Eufor pour le Darfour menacée par la guerre civile au Tchad, in: Le Monde, 02./03.12.2007.

31 Tschad war an der großen Antiterrorismus-Übung der USA in der Sahara 2005 beteiligt. Diese fand im Rahmen der Trans-Sahara Counter Terrorism Initiative statt, die von den USA 2005 für fünf Jahre initiiert wurde. Vgl. Martin Pabst: Die US-amerikanische Politik gegenüber Subsahara-Afrika, in: Österreichische Militärische Zeitschrift/ÖMZ 4/2006, S. 441; vgl. auch Catherine Fellows: US targets Sahara 'terrorist haven', in: BBC News, 08.08.2005.

32 AFRICOM hat den Auftrag, Operationen und Aktivitäten der US-Streitkräfte in Afrika zu stärken, die Fähigkeiten der afrikanischen Partner zu erweitern und ein neues Modell integrierter zivil-militärischer Zusammenarbeit zu entwickeln. Vgl. J. Stephen Morrison/Kathleen Hicks: AFRICOM Commentary, 5. Oktober 2007, Center for Strategic and International Studies, Washington, D.C., abrufbar unter: http://www.csis.org/media/ csis/pubs/071004_commentary_africom.pdf (letzter Zugriff: 25.01.2008). Vgl. auch die offizielle Seite des AFRICOM, abrufbar unter: http://www.africom.mil/ (letzter Zugriff: 12.03.2008). 
Wo es um Rohstoffe in Afrika geht, ist China nicht weit. So soll es Rebellen im Kampf gegen Déby mit Waffenlieferungen unterstützt haben. Daraufhin hat dieser 2006 die diplomatischen Beziehungen zu Taiwan abgebrochen und Verhandlungen mit Peking über die Exploration weiterer Ölfelder begonnen. China hat zudem Bohrlizenzen für mehrere Explorationszonen von einem kanadischen Unternehmen gekauft. Es wird auch die erste Ölraffinerie des Landes bauen. Für das Reich der Mitte, das bereits einen großen Teil des sudanesischen Öls abnimmt, ist der Zugang zu tschadischem Rohöl von großem Interesse. ${ }^{33}$ Das Öl könnte über die afrikanische Westküste oder - falls die Pipeline im Tschad mit dem im Sudan errichteten Netz verbunden würde - über das Rote Meer exportiert werden. Ein solches Vorhaben setzt allerdings eine Befriedung in Darfur und einen Ausgleich zwischen Tschad und Sudan voraus. Die jüngst an den Tag gelegte konziliantere Haltung Pekings im DarfurKonflikt und sein Engagement in der UNAMID können also auch auf seine Energieinteressen in beiden Ländern zurückgeführt werden. ${ }^{34}$

Die Interessenlage humanitärer Organisationen und Nichtregierungsorganisationen (NGOs) ist im Hinblick auf den Einsatz der EUFOR zwiespältig. Einerseits begrüßen ihn die meisten angesichts der sich dramatisch verschlechternden humanitären Lage. So richtete die ehemalige UN-Hochkommissarin für Menschenrechte und heutige Präsidentin der NGO ,Realizing Rights: The Ethical Globalization Initiative', Mary Robinson, am 11. Januar 2008 einen dramatischen Appell an die EU-Mitgliedstaaten, ausreichend Kräfte für EUFOR Tchad/RCA bereitzustellen und diese sofort zu entsenden. ${ }^{35}$ Auch humanitäre Organisationen wie Misereor oder der UNHCR unterstützen im Prinzip das EU-Engagement. Allerdings gibt es auch Befürchtungen, dass die Neutralität und Unparteilichkeit von EUFOR seitens der Konfliktparteien bezweifelt werden könnte und sich dadurch negative Konsequenzen für die humanitäre Arbeit ergeben könnten. ${ }^{36}$

\section{Herausforderungen und offene Fragen}

Wir haben es also mit einer Vielzahl von substaatlichen, staatlichen und internationalen Akteuren und Interessen sowie einer komplexen und dynamischen Abfolge von Aktivitäten zu tun. Das macht die Aufgabe für die Europäische Union nicht leichter. Die Union sieht sich also mit erheblichen Herausforderungen und einigen offenen Fragen konfrontiert:

Warum wurde die im Krisenmanagementkonzept geforderte Stärke von 4.000 Einsatzkräften nicht erreicht? Die Reaktion der Mitgliedstaaten auf den Truppenstellerkonferenzen war insgesamt verhalten. Bis Mitte Oktober 2007 wurden nur 2.500 Einsatzkräfte zugesagt, davon 1.500 aus Frankreich. Die französische Vorgabe lautete aber, dass mindestens die Hälfte aller Einsatzkräfte von anderen Ländern gestellt werden müsse. ${ }^{37}$ So sollte vermieden werden, dass EUFOR als französische Operation wahrgenommen werden könnte. Daraufhin deutete Paris an, dass 3.000 Soldaten ausreichen würden. Nach fünf Truppenstellerkonferenzen wurden schließlich

33 Vgl. Roy May/Simon Massey: Chad: Politics and Security, Writenet Report, März 2007, S. 12-15.

34 Vgl. Howard W. French/Lydia Polgreen: China, Filling the Void, Drills for Riches in Chad, in: The New York Times, 13.08.2007.

35 Vgl. Mary Robinson: Time is running out, in: International Herald Tribune, 11.01.2008.

36 Vgl. Integrated Regional Information Network (IRIN): Chad: Thoughts on the pending EU/UN mission from $\mathrm{UN}^{\text {‘ }}$ top humanitarian official, 17.12.2007, abrufbar unter: http://www.irinnews.org/PrintReport.aspx?ReportId=75906 (letzter Zugriff: 10.01.2008) und AllAfrica Global Media: Revised Plan for Peacekeeping Raises Concern, 23.08.2007, abrufbar unter: http://www.allafrica.com/stories/printable/200708230420.html (letzter Zugriff: 07.09.2007).

37 Interview mit dem Auswärtigen Amt am 12. September 2007. 
3.700 Einsatzkräfte zugesagt. ${ }^{38}$ Dazu trug vor allem die Bereitschaft Frankreichs bei, weitere 600 Soldaten zur Verfügung zu stellen. Pariser Hoffnungen auf weitere Kontingente blieben bislang unerfüllt. Insbesondere Deutschland signalisierte sehr zum französischen Leidwesen frühzeitig, dass es die Mission zwar politisch unterstützt, sich aber nicht mit Bodentruppen beteiligen wird. ${ }^{39}$ Während sich Paris enttäuscht über die bislang an den Tag gelegte deutsche Hartleibigkeit zeigte, verstand das offizielle Berlin den französischen Ärger nicht. Zu der Verstimmung beigetragen hat wohl auch die von Berlin und London betriebene Regelung, die ,gemeinsamen Kosten" 40 für die Operation auf 100 Millionen Euro zu beschränken. Schließlich wurden knapp 120 Millionen bewilligt, ${ }^{41}$ was einem Anteil von 15 Prozent an den Gesamtkosten entspricht. Folglich musste Frankreich tiefer in die Tasche greifen und einen Großteil der (wahrscheinlich viel zu niedrig) geschätzten 400-500 Millionen Euro selbst tragen. ${ }^{42}$

Reicht die personelle Stärke des Einsatzkontingents aus? Diese Frage ist schwer zu beantworten, weil es keinen einheitlichen und immer gültigen Maßstab für die Bestimmung der für eine bestimmte Militäroperation notwendigen Truppenstärke gibt. Diese hängt von verschiedenen Faktoren ab, etwa der Art der Operation, dem Auftrag und der Größe des Operationsgebiets. Legt man gängige Maßstäbe für Stabilisierungseinsätze zugrunde, so sind vier bis zehn Soldaten pro tausend Einwohner erforderlich. ${ }^{43}$ Bei einer Bevölkerung von 1,25 Millionen einschließlich der Flüchtlinge aus Darfur und der Binnenvertriebenen im Operationsgebiet müsste EUFOR Tchad/RCA also 5.000 bis 12.500 Einsatzkräfte umfassen. ${ }^{44}$ Der bisherige Ansatz im Krisenmanagementkonzept der Europäischen Union von 4.000 Einsatzkräften läge demnach zu niedrig. ${ }^{45}$ Zudem sprechen sowohl die Größe des Einsatzgebiets (ungefähr $300.000 \mathrm{~km}^{2}$ ) als auch die völlig unterentwickelte Transportinfrastruktur für eine dichtere Präsenz, es sei denn, die fehlende Personalstärke würde durch größere Luftbeweglichkeit ausgeglichen.

Ist die Europäische Union in der Lage, über ein ganzes Jahr die sehr anspruchsvollen Fähigkeiten bereitzustellen, die angesichts der Größe des Einsatzgebietes und der besonderen An-

38 Irland stellt mit 400 Einsatzkräften das zweitstärkste Kontingent und wird die Operation vom Hauptquartier Mont Valérien bei Paris aus leiten. Frankreich, das großen Wert auf den multinationalen Charakter der ESVPMission legt, stellt den Force Commander. Zusagen gaben ferner Polen (400), Schweden (200), Österreich (180), Rumänien (150), Belgien (120), die Niederlande (70), Spanien (70), Finnland (60), Italien (60), Portugal (30), Griechenland (20), Slowenien (15). Vgl. Europolitics 3455, 24. Januar 2008, S. 16-17.

39 Berlin hat vier Stabsoffiziere in das Hauptquartier entsandt. Vgl. Frankfurter Allgemeine Zeitung: Zusagen für Tschad-Truppe der EU, 01.10.2007.

40 Die ,gemeinsamen Kosten“ der militärischen Aktivitäten im Rahmen der ESVP, die von allen Teilnehmerstaaten gemäß dem Athena-Mechanismus anteilsmäßig zu tragen sind, decken nur einen kleinen Teil der Gesamtkosten. Den größten Anteil der operativen Ausgaben müssen dagegen die teilnehmenden Nationen nach dem Prinzip ,costs lie where they fall“ tragen. Vgl. Rat der Europäischen Union: Athena, abrufbar unter: http://consilium.europa.eu/cms3_fo/showPage.asp?id=746\&lang=EN\&mode=g (letzter Zugriff: 12.03.2008).

41 Zunächst muss eine Kostenschätzung für die Verabschiedung der Gemeinsamen Aktion abgegeben werden. Danach erfolgt die detaillierte militärische Ausplanung durch den Befehlshaber, die in der Regel zu einem höheren Ansatz führt, der dem sogenannten Athena-Ausschuss zur Bewilligung vorgelegt werden muss.

42 Vgl. Philippe Bernard/Laurent Zecchini: Paris financera l'essentiel de l'opération Tchad-Centrafrique, in: Le Monde, 5.10.2007 und Philippe Bernard/Philippe Ricard: La force européenne en Centrafrique et au Tchad sera à dominante française, in: Le Monde, 12.01.2008. Stimmt der in Le Monde (Laurent Zecchini: L'Union européenne lance l'opération Eufor au Tchad et en Centrafrique) vom 30.01.2008 angegebene Anteil von 15 Prozent „gemeinsamer Kosten“ an den Gesamtkosten, so müssten sich Letztere auf über 700 Mio. Euro belaufen.

43 Vgl. Bjoern H. Seibert: African Adventure? Assessing the European Union's Military Intervention in Chad and in the Central African Republic, MIT Security Studies Programme Working Paper, November 2007, S. 31-33.

44 Die Planungen der UNO gehen von zwei Optionen aus: A) Eine Infanteriebrigade von 6.000 Soldaten, 20 Hubschraubern und ein Beobachtungsflugzeug; B) eine Infanteriedivision von 10.900 Soldaten, elf Hubschraubern und zwei Beobachtungsflugzeugen. Vgl. UN-Sicherheitsrat: Report of the Secretary General on Tchad and the Central African Republic, S/2007/97, 23. Februar 2007, S. 13-14.

45 Die EU hatte ursprünglich drei Optionen für die Personalstärke der EUFOR erwogen: Option A) 3.000, Option B) 4.000, Option C) 8.000 Einsatzkräfte. 
forderungen des Geländes notwendig sind? Immerhin soll sich der Verband aus einer luftbeweglichen Komponente, Einheiten mit großer taktischer Beweglichkeit und einer starken Aufklärungskomponente zusammensetzen. Es handelt sich hierbei um Einsatzmittel, die knapp und wertvoll sind. So fehlen Hubschrauber mit spezifischen Fähigkeiten sowohl der ISAF in Afghanistan als auch der UNAMID im Sudan. Paris hat diese Lücke mit weiteren Hubschraubern und einem taktischen Transportflugzeug partiell verkleinert. ${ }^{46}$ Eine weitere Zusage über die Teilnahme mit Hubschraubern kam Anfang März von Moskau, die Verhandlungen über weitere Beiträge etwa von Norwegen und der Ukraine liefen zu diesem Zeitpunkt noch. Gleichwohl bleiben die logistischen Herausforderungen für die bislang größte Militäroperation der Europäischen Union in Afrika groß. Da von der host nation keine nennenswerte Unterstützung zu erwarten ist, müssen der Unterhalt der Truppe, also die Versorgung mit Gütern wie Wasser, Treibstoff, Nahrung und Unterkünften sowie der militärische Nachschub größtenteils auf dem Luft- und Seeweg sichergestellt werden. Das ist langwierig und teuer ${ }^{47}$ Insofern ist die Verärgerung in Paris, das nicht nur die militärische Hauptlast des Einsatzes trägt, sondern auch den größten Teil der Logistik übernimmt, nachvollziehbar. Weil anspruchsvolle Fähigkeiten über einen längeren Zeitraum vorgehalten werden müssen, sieht sich die Europäische Union gezwungen, den Prozess der Kräftegenerierung auch nach dem Beginn der Stationierung von EUFOR fortzusetzen. ${ }^{48}$ Es bleibt abzuwarten, ob diese Bemühungen Erfolg haben werden.

Wie neutral und unparteiisch wird sich EUFOR Tchad/RCA verhalten können? Störfeuer sind sowohl vom Präsidenten des Tschad als auch von den verschiedenen Rebellengruppen und Milizen zu erwarten. Idriss Déby hat in der Affäre um die Hilfsorganisation ,Arche de Zoé' gezeigt, dass er geschickt mit Stimmungen in der Bevölkerung umzugehen weiß.49 $\mathrm{Zu}-$ dem hat er die EUFOR-Mission zunächst nur widerstrebend akzeptiert. Einerseits konnte er die dringende Bitte der UNO und Frankreichs nicht ablehnen, andererseits steht für ihn der Kampf gegen die Rebellen im Vordergrund. Die Intensivierung der Kämpfe seit Herbst 2007 führte zu Bombenangriffen der tschadischen Luftwaffe auf feindliche Rebellen im Sudan und zu einem weiteren Umsturzversuch im Tschad. Diesen nutze Déby wiederum zu einem Schlag gegen die zivile Opposition, wodurch der ein Jahr zuvor begonnene Demokratisierungsprozess möglicher Weise gestoppt wurde. Offiziell handelt es sich bei EUFOR Tchad/ RCA um eine humanitäre Intervention, durchgeführt von neutralen und unparteiischen Kräften. Paris unterstützt aber auch den Präsidenten des Tschad, etwa durch die Drohung, zur Not militärisch gegen die Rebellen vorzugehen. ${ }^{50}$ Diese Drohung erfolgte allerdings erst, nachdem die AU das Vorgehen der Rebellen verurteilt hatte. Der UNO-Sicherheitsrat rief kurz darauf zur Unterstützung der tschadischen Regierung auf, jedoch ohne die von Paris gewünschte Warnung an den Sudan und ohne den Zusatz ,,mit allen zu Verfügung stehenden Mitteln“.51

46 Insgesamt waren ursprünglich 20 bis 30 Hubschrauber vorgesehen. Die EU-Staaten Frankreich (9), Polen (2) und Rumänien (2) stellen 13 Hubschrauber. Vgl. Frankfurter Allgemeine Zeitung: Füllt Paris die Lücken in der Tschad-Truppe?, 11.01.2008 und Frankfurter Allgemeine Zeitung: Russland verspricht Hubschrauber für Tschad-Einsatz 12.01.2008 sowie Europolitics 3455, 24. Januar 2008, S. 16.

47 So soll alleine der Lufttransport eines Fünftel der Fracht für die Stationierung zwischen 9,2 und 16,9 Mio. US Dollar kosten. Vgl. Seibert: African Adventure?, 2007, S. 24.

48 Mission militaires de UE, in: Europolitique 3484, 5. März 2008, S. 13.

49 Im Oktober 2007 wurden 16 Europäer, darunter neun Franzosen, im Tschad der Kindesentführung angeklagt und erst nach der persönlichen Intervention von Präsident Sarkozy entlassen. Vgl. Alain Barluet: Paris embarassé met L'Arche de Zoé au pilori, in: Le Figaro, 30.10.2007.

50 Es soll auch zu Gefechten zwischen französischen Soldaten und Rebellen am Flughafen von N'Djamena gekommen sein. Vgl. Frankfurter Allgemeine Zeitung: Rebellen bieten Waffenstillstand an, 06.02.2008.

51 Der Sudan will nach Pariser Lesart die Stationierung der EUFOR ebenso hintertreiben wie die der UNAMID. Vgl. Le Monde: L'armée d'Idriss Déby a résisté à l'offensive rebelle sur N'Djamena, 05.02.2008; und Frankfurter Allgemeine Zeitung: Rebellen: taktischer Rückzug, 05.02.2008. 
Während die Europäische Union hofft, dass der EUFOR-Einsatz eine beruhigende Wirkung auf die Aktivitäten der Rebellen im Einsatzgebiet Tschad/ZAR haben wird, unterstützt Paris die Armee des Tschad insbesondere in den Bereichen Luftaufklärung und Logistik. Die Rebellen haben nach den jüngsten Luftangriffen der tschadischen Streitkräfte, die ihnen schwere Verluste beigebracht haben sollen, erklärt, man befinde sich mit Frankreich im Kriegszustand. Es ist also zu bezweifeln, dass die Versicherung von Verteidigungsminister Hervé Morin, er schließe einen Präventivschlag der französischen Armee gegen die Rebellen aus, deren negative Wahrnehmung verändert hat. ${ }^{52}$ Allerdings haben sich die französischen Einheiten während des jüngsten Umsturzversuches erstaunlich lange zurückgehalten. Drei Gründe dürften dafür ausschlaggebend gewesen sein: Erstens hat Präsident Sarkozy die Devise ausgegeben, nur noch mit internationalem Mandat in Afrika zu intervenieren. ${ }^{53}$ Zweitens sollte die gerade angelaufene Stationierung der EUFOR nicht gefährdet werden ${ }^{54}$ und drittens sollte den Akteuren vor Ort wohl auch verdeutlicht werden, dass Paris der entscheidende Machtfaktor bleibt. ${ }^{55}$ Gleichwohl dürfte es sehr schwer sein, die Akteure von der Neutralität der EUFOR zu überzeugen. ${ }^{56}$

Was passiert, wenn die UNO-Blauhelmtruppe, die das EU-Kontingent ablösen soll, nicht pünktlich oder gar nicht erscheint? Einen entsprechenden Beschluss kann der UNOSicherheitsrat heute noch nicht fassen. Die Lage im Krisengebiet kann sich aber ebenso ändern wie die politische Stimmung im Sicherheitsrat und in den vor Ort engagierten EUStaaten selbst. Fraglich ist auch, ob Präsident Déby einer militärischen UNO-Mission dieses Mal zustimmen wird. Auf jeden Fall ist zu erwarten, dass die komplexe humanitäre (und die sie verursachende politische) Krise nicht innerhalb eines Jahres gelöst sein wird. Es ist wahrscheinlich, dass es sich um ein internationales Engagement handeln wird, dessen Ende nicht absehbar ist. Folglich muss die Europäische Union den Einsatz von EUFOR entweder verlängern, was aber aus finanziellen und politischen Gründen nur sehr schwer möglich sein dürfte. Oder sie muss rechzeitig von einer UN-Truppe abgelöst werden, wie es gemäß dem Krisenmanagementkonzept der Union vorgesehen ist. Es bestehen allerdings Zweifel, ob New York dazu in der Lage sein wird. Die UNO ist mit entsprechenden Einsätzen bereits überdehnt. Sie hat über 80.000 Blauhelme weltweit im Einsatz und bereits Schwierigkeiten die Hybridmission für Darfur entsprechend auszustatten. ${ }^{57}$ Über weit projizierbare einsatz- und durchhaltefähige Kräfte verfügen nur wenige Staaten, wie zum

52 Vgl. Thomas Scheen: Kämpfe an allen Fronten, in: Frankfurter Allgemeine Zeitung, 07.12.2007.

53 Vgl. Philippe Bernard/Natalie Nougayrède: Tchad: Paris avait décidé de ne pas intervenir, in: Le Monde 08.02.2008, S. 4.

54 Die Stationierung wurde zeitweise suspendiert. EUFOR Tchad/RCA ist laut Verteidigungsminister Hervé Morin notwendiger denn je. Vgl. Hervé Morin: „Nous tenons nos engagements“, Interview mit Hervé Morin, in: Le Figaro, 03.02.2008.

55 Die aus insgesamt rund 300 Pickups bestehenden Wagenkolonnen der 2.000 Rebellen mussten 700 km zurücklegen und wären eigentlich leichte Ziele für Luftangriffe gewesen. Frankreich soll Déby nur angeboten haben ihn auszufliegen. Vgl. Alain Barluet: Paris à volé au secours de la victoire d'Idriss déby, abrufbar unter: http:// www.lefigaro.fr/international/ (letzter Zugriff: 13.03.2008).

56 Dies wird durch die erste Kampfhandlung bestätigt, bei der ein EUFOR-Soldat verwundet und ein weiterer gefangengenommen wurde. Vgl. International Herald Tribune: EU soldiers are shot at in Sudan, 05.03.2008.

57 UNAMID soll 20.000 Soldaten und 6.000 Polizisten umfassen. Die Dislozierung hat zwar Anfang Januar 2008 offiziell begonnen. Die Operation leidet aber an fehlender Mobilität und Kampfkraft (es fehlen 18 Transporthubschrauber und sechs Kampfhubschrauber) sowie mangelhaften logistischen Fähigkeiten. Zudem erschwert die Regierung des Sudan das Vorhaben, indem sie etwa Norwegen und Schweden nicht erlaubte, 350 Pioniere zu entsenden, keine Nachtflüge zulässt, Unterstützungsmaßnahmen verweigert oder gar UN-Konvois militärisch „testen“, d.h. beschießen lässt. Angesichts dieser Lage warnte Jean-Marie Guéhenno den UN-Sicherheitsrat bereits vor einem Scheitern der Mission. Vgl. Philippe Bolopion: Au Darfour, ,un manque tragique de moyens essentiels" affaiblit la force commune ONU-UA, in: Le Monde, 11.01.2008. 
Beispiel die USA und Kanada. Diese zeigen aber kein Interesse an einem militärischen Engagement im Tschad. Eine Folgemission müsste sich also aus den Entwicklungsländern rekrutieren. Aufgrund der unzureichenden Mobilität müsste diese Truppe wesentlich größer sein (mindestens 10.000 Soldaten) und würde auf erheblich größere Versorgungsprobleme stoßen als EUFOR. Des Weiteren stellt sich die Frage der Finanzierung des Einsatzes angesichts bereits existierender Belastungen wie etwa in der Demokratischen Republik Kongo, wo die UN-Friedensmission MONUC bereits mehr als eine Milliarde US-Dollar pro Jahr kostet, und des angelaufenen Einsatzes der Hybridtruppe in Darfur, der noch teurer wird. Schließlich ist der Zeitkorridor für eine Folgetruppe extrem eng. Soll EUFOR im März 2009 abgelöst werden, bleiben nach dem Ende der Regenzeit fünf Monate. Auf jeden Fall müsste das UN-Mandat vorher verabschiedet und die Frage der Truppensteller sowie der Ausrüstung noch 2008 geklärt werden. ${ }^{58}$

Dass die Europäische Union der Sache selbst nicht ganz traut ist daran zu erkennen, dass nach sechs Monaten die Wirkung des europäischen Einsatzes auf die humanitäre und die Sicherheitslage überprüft werden soll. Das ist ein löbliches Unterfangen, nur soll diese Überprüfung anhand von Indikatoren der UNO erfolgen, die noch nicht feststehen. Bleibt nur zu hoffen, dass das offiziell angestrebte Ziel (,endstate') - die vollständige Stationierung der Polizeikräfte und des Einsatzverbandes der UNO sowie die Umsetzung von Wiederaufbauund Hilfsprogrammen für die freiwillige Rückkehr der Vertriebenen - nach spätestens zwölf Monaten erreicht und der demokratische Prozess im Tschad wieder aufgenommen werden wird. Zwei negative Szenarien sind aber ebenso denkbar: erstens die Verwicklung von EUFOR in den tschadischen Bürgerkrieg mit nicht absehbaren politischen Folgen und zweitens die Wahl zwischen den Übeln, entweder den Tschad sudanesischem Einfluss zu überlassen oder die Alleinherrschaft von Idriss Déby zu stützen.

\section{Rationalistisches Kalkül, humanitärer Idealismus oder Logik der Angemessenheit?}

Angesichts der oben skizzierten Schwierigkeiten stellt sich die Frage, warum die Europäische Union und ihre Mitgliedstaaten die Operation EUFOR Tchad/RCA durchführen. Folgen sie eher einer rationalistischen (im Sinne von zweckrational) und interessenfixierten Logik, einer idealistischen Ethik des Guten oder einer Logik der Angemessenheit, die Ziele und Mittel, Normen und Werte, Interessen und ,glokale ${ }^{\text {"59 }}$ Kontexte in friedenspolitischer Absicht verbindet? Für den Einsatz können eine Reihe rationalistischer Argumente angeführt werden wie Einflusssicherung in der Region, Stabilisierung des Tschad und der ZAR, Energiesicherheit, Stärkung der ESVP, aber auch Stärkung der Rolle Frankreichs oder einfach persönliche Ambitionen des französischen Präsidenten. Rationalistische Gegenargumente weisen auf die Gefahren und Kosten des Engagements hin oder auf das mangelnde Interesse einzelner EU-Staaten, sich vor den Karren der Pariser Afrikapolitik spannen zu lassen.

Gleichzeitig spielen normative Erwägungen eine signifikante Rolle, ohne sich jedoch auf einen bloßen Idealismus zu beschränken. So ist die humanitäre Notlage in der Region unbestritten. Ihre enge Verknüpfung mit dem Darfur-Konflikt setzt die internationale Gemeinschaft unter Handlungsdruck. Der Entsendung von EUFOR Tchad/RCA gingen gescheiterte

58 Da die Initial Operational Capability voraussichtlich Mitte März 2008 erreicht wird und die Bedarfsplanung für die Folgemission sechs Monate später erfolgen soll, müssen diese Fragen zwischen Oktober 2008 und Februar 2009 geklärt werden. Vgl. Rat der Europäischen Union: Council Conclusions on Sudan/Chad/Central African Republic, 2846th External Relations Council meeting, 28. January 2008.

59 „Glokal“ bezeichnet den engen Zusammenhang von lokalen und globalen Ereignisse und Prozessen. Vgl. dazu Ulrich Beck: Was ist Globalisierung?, Frankfurt am Main 1998. 
Bemühungen der UNO, eine Blauhelmtruppe zu entsenden, und des französischen Außenministers Bernard Kouchner, humanitäre Korridore vom Tschad nach Darfur einzurichten, voraus. Die enge Kooperation mit der UNO entspricht dem EU-Ziel eines effektiven Multilateralismus und basiert auf der Gemeinsamen Erklärung über die Zusammenarbeit im Bereich Krisenmanagement in Afrika von 2003. ${ }^{60}$ Beide Sicherheitsorganisationen verstehen sich als friedenspolitisch engagierte „Normenunternehmer“ mit besonderer Verantwortung für Afrika und die Umsetzung der Millennium Development Goals. ${ }^{61}$ Diese Ziele sind nur zu erreichen, wenn zentrale Krisenherde befriedet werden. Dazu gehört auch das Krisendreieck Sudan/Tschad/ZAR. Die Tatsache, dass sich mit Frankreich nur ein Mitglied der ,EU-3 im Operationsgebiet beteiligt, kann allerdings als Indiz für besondere nationale Interessen angeführt werden. Das starke Engagement der neutralen und paktungebundenen EU-Mitglieder spricht wiederum ebenso für die Relevanz normativer Präferenzen wie die insgesamt große Beteiligung von 14 EU-Mitgliedstaaten vor Ort und 22 im Hauptquartier. ${ }^{62}$ Die meisten von ihnen verfolgen keine signifikanten geo-, macht- oder energiepolitischen Interessen in der Region, sondern orientieren ihre Außenpolitik eher an friedens- und entwicklungspolitischen Zielen. Das bedeutet jedoch nicht zwangsläufig, dass es sich um selbstlose Ziele handelt. Sowohl für Staaten als auch für kollektive Akteure wie die Europäische Union kann die Aufrechterhaltung und der Transfer bestimmter Normen - etwa durch die humanitäre Intervention der EUFOR Tchad/RCA - von stabilitäts- und sicherheitspolitischer Relevanz, also in ihrem aufgeklärten Eigeninteresse sein. ${ }^{63}$

Gleichwohl zeigen die großen Probleme bei der Zusammenstellung, Stationierung und Durchführung der EUFOR Tchad/RCA wie schwierig es ist, die vielfältigen Ziele, Interessen, Werte und Normen in einem konkreten Fall gemäß einer Logik der Angemessenheit miteinander in Einklang zu bringen. Immerhin dauerte der gesamte Entscheidungsprozess sechs Monate und es waren nicht weniger als fünf Truppenstellerkonferenzen nötig, was bei einer humanitären Notlage eigentlich untragbar ist. Des Weiteren deutet die widersprüchliche Reaktion auf die Verhaftung führender tschadischer Oppositioneller auf unterschiedliche Präferenzen und gravierende Zielkonflikte der Akteure hin. Während Entwicklungskommissar Louis Michel die sofortige Freilassung der Oppositionellen verlangte, verzichtete Paris zunächst auf eine öffentliche Verurteilung. ${ }^{64}$ Zudem besteht für die Mission immer noch eine riskante Diskrepanz zwischen Zielen und Mitteln. Die wesentlichen Gründe für diese Probleme sind mangelnder politischer Wille und eine inkonsistente politische Handlungslogik, unzureichende Fähigkeiten und fehlender politischer Rückhalt in der Öffentlichkeit.

60 Vgl. Europäische Union/Vereinte Nationen: Joint Declaration on UN-EU Co-operation in Crisis Management, 24. September 2003, abrufbar unter: http://www.europa-eu-un.org/articles/en/article_2768_en.htm (letzter Zugriff: 29.01.2008)

61 Ein Normenunternehmer kann definiert werden als ein Akteur, ,that sets out to change the behavior of others“. Ann Florini: The Evolution of International Norms, in: International Studies Quarterly 3/1996, S. 375 . Vgl. auch Simon Rushton: The UN Secretary-General and Norm Entrepreneurship: Boutros-Ghali and Democracy Promotion, in: Global Governance 1/2008, S. 95-110.

62 Nur Bulgarien, Dänemark, Estland, Lettland und Malta beteiligen sich nicht an EUFOR Tchad/RCA.

63 Vgl. dazu Albrecht Schnabel: Humanitarian Affairs and State Foreign Policy, Paper presented at the annual meeting of the International Studies Association, Hilton Hawaiian Village, Honolulu, Hawaii, März 2005, abrufbar unter: http://www.allacademic.com/meta/p70264_index.html (letzter Zugriff: 17.03.2008).

64 Vgl. Natalie Nougyrède/Jean Pierre Tuquoi: Paris embarrassé par les arrestation d'opposants tchadiens au régime d'Idriss Déby, in: Le Monde, 13.02.2008. Sarkozys späterer Versuch, die verschleppten Oppositionellen während seines Kurzaufenthaltes im Tschad frei zu bekommen, schlug fehl. Vgl. Philippe Bernard: A N'Djamena, M. Sarkozy demande «la vérité» sur les opposants disparus, in: Le Monde, 29.02.2008. 
Die EU-Mitglieder müssen also erstens ihre bisherigen Anstrengungen zur Formulierung und Umsetzung einer gemeinsamen Afrikapolitik mit dem Ziel intensivieren, zu einer gemeinsamen Handlungslogik zu kommen, die den proklamierten Prinzipien und Normen besser entspricht und zugleich in operative Ziele herunter gebrochen werden kann. ${ }^{65}$ Ansonsten wird der Verdacht genährt, dass luftige Prinzipien und Normen zwar deklariert werden, in der Praxis aber kurzfristige Eigeninteressen vorgehen. Zweitens sollten die vielfältigen Fähigkeitslücken geschlossen werden, um angemessen agieren zu können. Durch die Stärkung und die funktionale Vernetzung analytisch-planerischer, finanzieller, administrativer, entwicklungspolitischer, polizeilicher und militärischer Fähigkeiten würde auch die eigene Glaubwürdigkeit erhöht. ${ }^{66}$ Drittens muss sich die Europäische Union die Unterstützung ihrer Bürgerinnen und Bürger durch eine transparentere Sicherheitspolitik erarbeiten. Nötig sind also weniger Geheimniskrämerei oder Schönrederei und mehr öffentlicher Diskurs über das Für und Wider des EU-Krisenmanagements in Afrika. Dabei muss in jedem Einzelfall erund geklärt werden, ob sich Normen und Interessen sowie Ziele und Mittel angemessen zueinander verhalten. Im Falle der Operation EUFOR Tchad/RCA bestehen daran erhebliche Zweifel.

\begin{tabular}{|ll|}
\hline AFRICOM & Regionalkommando der US-Streitkräfte für Afrika \\
AMIS & - African Union Mission in the Sudan \\
EUFOR TCHAD/RCA - & militärische Überbrückungsoperation der EU zur Unterstützung der multi- \\
& dimensionalen MINURCAT in der Republik Tschad und der Zentralafrika- \\
& nischen Republik \\
EUPOL Kinshasa & - Polizeimission der EU in Kinshasa (Demokratische Republik Kongo) \\
EUPOL RD Congo & - Beratungs- und Unterstützungsmission der EU im Zusammenhang mit der \\
& Reform des Sicherheitssektors in der Demokratischen Republik Kongo \\
EU SSR Guinea-Bissau - & Beratungs- und Unterstützungsmission der EU im Zusammenhang mit der \\
& Reform des Sicherheitssektors in Guinea-Bissau \\
FOMUC & Force multinationale en Centrafrique de la CEMAC (Communauté Écono- \\
& mique et Monétaire de l'Afrique Centrale) \\
ISAF & International Security Assistance Force, Afghanistan \\
MINURCAT & - United Nations Mission in the Central African Republic and Chad \\
MONUC & United Nations Organization Mission in the Democratic Republic of the \\
& Congo \\
UNAMID & African Union/United Nations Hybrid operation in Darfur
\end{tabular}

65 Vgl. auch Sibylle Schneipers/Daniela Sicurelli: Empowering Africa: normative power in EU-Africa relations, abrufbar unter: http://www.unc.edu/euce/eusa2007/sicurelli-d-05i.pdf (letzter Zugriff: 17.03.2008).

66 Vgl. dazu Hans-Georg Ehrhart: The EU as a civil-military crisis manager. Coping with internal security governance, in: International Journal, Spring 2006, S. 433-450. 